\title{
Operative Treatment of Intra-Articular Distal Radius Fractures With versus Without Arthroscopy: study protocol for a randomised controlled trial
}

Marjolein A. M. Mulders ${ }^{1 *}$, Caroline A. Selles ${ }^{1}$, Joost W. Colaris ${ }^{2}$, Rolf W. Peters ${ }^{1}$, Mark van Heijl', Berry I. Cleffken ${ }^{3}$ and Niels W. L. Schep ${ }^{3}$

\begin{abstract}
Background: In the past several years, an increase in open reduction and internal fixation (ORIF) for intra-articular distal radius fractures has been observed. This technique leads to a quicker recovery of function compared to non-operative treatment. However, some patients continue to have a painful and stiff wrist postoperatively. Arthroscopically assisted removal of intra-articular fracture haematoma and debris may improve the functional outcomes following operative treatment of intra-articular distal radius fractures. The purpose of this randomised controlled trial is to determine the difference in functional outcome, assessed with the Patient-Rated Wrist Evaluation (PRWE) score, after ORIF with and without an additional wrist arthroscopy in adult patients with displaced complete articular distal radius fractures.
\end{abstract}

Methods: In this multicentre trial, adult patients with a displaced complete articular distal radius fracture are randomised between ORIF with an additional wrist arthroscopy to remove fracture haematoma and debris (intervention group) and conventional fluoroscopic-assisted ORIF (control group). The primary outcome is functional outcome assessed with the PRWE score after three months. Secondary outcomes are wrist function assessed with the Disability of the Arm, Shoulder and Hand (DASH) score, postoperative pain, range of motion, grip strength, complications and cost-effectiveness. Additionally, in the intervention group, the quality of reduction, associated ligamentous injuries and cartilage damage will be assessed. A total of 50 patients will be included in this study.

Discussion: Although ORIF of intra-articular distal radius fractures leads to a quicker resume of function compared to non-operative treatment, some patients continue to have a painful and stiff wrist postoperatively. We hypothesise that, due to the removal of fracture haematoma and debris by an additional arthroscopy, functional outcomes will be better compared to the non-arthroscopically treated group.

Trial registration: ClinicalTrials.gov, NCT02660515. Registered on 13 January 2016.

Keywords: Distal radius fracture, Articular, Displaced, Wrist arthroscopy, Wrist function, PRWE, Ligamentous injuries, Randomised controlled trial

\footnotetext{
* Correspondence: m.a.mulders@amc.nl

${ }^{1}$ Trauma Unit, Department of Surgery, Academic Medical Center, University

of Amsterdam, P.O. Box 22660, 1100 DD Amsterdam, The Netherlands

Full list of author information is available at the end of the article
} 


\section{Background}

In the last decade, an increase in open reduction and internal fixation (ORIF) for distal radius fractures has been observed [1-3]. In particular, intra-articular distal radius fractures, which comprise almost 50\% of all fractures [4], are increasingly being treated operatively. This technique leads to a quicker resume of function in the first three to six months compared to non-operative treatment $[5,6]$. However, some patients continue to have a painful and stiff wrist postoperatively. Arthroscopically assisted removal of intra-articular fracture haematoma and debris may improve the functional outcomes following operative treatment of intra-articular distal radius fractures [7, 8]. Moreover, during arthroscopy the quality of the reduction $[7,9,10]$ and the presence of associated ligamentous injuries can be assessed [11-14].

Lindau et al. already examined the frequency of associated chondral and ligament lesions with arthroscopy in 50 patients in 1997 [14]. They described 35 subchondral haematomas in 16 cases, and an incidence of chondral lesions of approximately 33\%. These lesions may lead to the development of osteoarthritis in the long term [15]. Additionally, 98\% of the patients had a ligamentous injury. However, they found no major instability in these patients and it is uncertain if these injuries will be clinically relevant in the long term [16].

Although, no advantage of arthroscopically guided reduction over conventional fluoroscopic-assisted reduction in regard to functional and radiographic outcomes was found [17], to our knowledge no studies have been carried out to further examine the use of arthroscopy after ORIF to remove fracture haematoma and debris on functional outcomes. We hypothesise that, due to the removal of fracture haematoma and debris, functional outcomes will be better compared to the nonarthroscopically treated group. Therefore, the purpose of this randomised controlled trial (RCT) is to determine the difference in functional outcome, assessed with the Patient-Rated Wrist Evaluation (PRWE) score, after ORIF with and without an additional wrist arthroscopy in adult patients with displaced complete articular distal radius fractures. Furthermore, we aim to determine the difference in functional outcomes with the Disability of the Arm, Shoulder and Hand (DASH) score, postoperative pain, range of motion (ROM), grip strength, complications, and cost-effectiveness. Additionally, the quality of reduction, associated ligamentous injuries and cartilage damage will be assessed by arthroscopy.

\section{Methods/Design}

\section{Study objectives}

The primary objective is to determine the difference in functional outcome of ORIF with or without an additional arthroscopy to remove the fracture haematoma and debris in adult patients with displaced complete articular distal radius fractures (AO/OTA type $\mathrm{C}$ ).

The secondary objectives are to assess if additional wrist arthroscopy leads to less postoperative pain, a better ROM and grip strength, and fewer complications. Additionally, cost-effectiveness for both treatments is determined. Moreover, for patients undergoing additional wrist arthroscopy, the quality of reduction, associated ligamentous injuries and cartilage damage will be assessed.

\section{Study design}

The RADAR (Operative Treatment of Intra-Articular Distal Radius Fractures With versus Without Arthroscopy) trial is designed as a multicentre RCT, with a 1:1 allocation ratio and a superiority framework. Patients are randomised between ORIF with an additional wrist arthroscopy to remove fracture haematoma and debris (intervention group) and conventional fluoroscopic-assisted ORIF (control group). A total of three centres in the Netherlands are involved in recruiting patients (Additional file 1).

The design of the trial is compliant with the Standard Protocol Items: Recommendations for Interventional Trials [18] (Additional file 2).

\section{Study population}

The study population will consist of all adult patients who are diagnosed with a complete articular distal radius fracture (AO/OTA type $\mathrm{C}$ ) where the treating surgeon deems ORIF necessary. Independent radiologists will assess and classify complete articular distal radius fracture based on radiography according to the AO/OTA classification of fractures. All patients undergo a computed tomography $(\mathrm{CT})$ scan of the wrist. This is standard care in decision-making and planning for surgery [19].

\section{Inclusion criteria}

- Patients aged 18 years and older

- Displaced complete articular distal radius fracture (AO/OTA type C) as classified on lateral, posterioranterior, and lateral carporadial radiographs by a radiologist or trauma surgeon, requiring ORIF. An additional dorsal approach is allowed only when the dorsal capsule is not opened and thus leaving the radiocarpal joint untreated.

- Inacceptable alignment on radiograph defined, according to the Dutch National Guidelines [19], as:

$\bigcirc$ radial inclination $<15^{\circ}$;

$\bigcirc$ radial length (distance between lateral most radial tip and ulnar surface) $\leq 5 \mathrm{~mm}$;

$\bigcirc$ volar angulation $\geq 20^{\circ}$ or dorsal angulation $\geq 15^{\circ}$;

$\bigcirc$ articular step-off or gap $\geq 2 \mathrm{~mm}$. A gap is

defined as loss of articular congruity of the distal 
radius parallel to the articular surface and a stepoff perpendicular to the articular surface [20].

\section{Exclusion criteria}

- Dorsal plate fixation in case the radiocarpal joint needs to be opened

- Multiple trauma patients (Injury Severity Score (ISS) $\geq 16$ )

- Open distal radius fractures

- Other fractures in the ipsilateral extremity (except for a fracture of the ulnar styloid process)

- Fracture of the contralateral wrist (distal radius, distal ulna or one of the carpal bones)

- Patients with impaired wrist function before injury due to arthrosis, rheumatoid arthritis, neurological disorders or malunion of the upper limb or patients suffering from disorders of bone metabolism other than osteoporosis (i.e. Paget's disease, renal osteodystrophy, osteomalacia) or connective tissue disease or (joint) hyperflexibility disorders such as Marfan's or Ehler Danlos

- Patients with insufficient comprehension of the Dutch language to understand the study information and informed consent process, the rehabilitation program and other treatment information as judged by the attending physician

\section{Interventions}

All patients will be treated by a certified (orthopaedic) trauma surgeon with experience in ORIF of distal radius fractures and wrist arthroscopy. In both groups ORIF of the distal radius fracture will be similar. The intervention group will be treated with wrist arthroscopy following ORIF. A delay of at least five days before performing arthroscopy is mandatory to enable visualisation due to the organisation of the hematoma. The operation has to be performed within three weeks after the initial trauma.

Antibiotic prophylaxis (Cefazoline, $1000 \mathrm{mg}$ i.v.) is given preoperatively, according to the current standard. The volar approach according to Henry will be used [21]. This entails an incision between the radial artery and the tendon of the flexor carpi radialis. The pronator quadratus muscle from will be detached from its distal and lateral side and lifted for optimal exposure to the fracture site. After the fracture site is revealed, the fracture will be debrided, reduced and fixated with an appropriate volar locking plate. The type and brand of the plate are at the discretion of the treating surgeon. When a dorsal approach is deemed necessary the distal radius will be approached through the third dorsal extensor tendon compartment, without opening the dorsal capsule. Fluoroscopic images are obtained to evaluate the quality of articular reduction. Wrist arthroscopy will be performed when the treating surgeon is satisfied with the result of the ORIF.

During wrist arthroscopy, the forearm will be positioned upright and in neutral position, the elbow flexed by $90^{\circ}$ and axial traction of 4-6 kg will be performed. Four portals are created dorsally by superficial stab incisions and blunt preparation through the joint capsule; one midcarpal radial (MCR) and ulnar (MCU) portal and one radiocarpal 3-4 and 6-R portal (Fig. 1). Portals may be changed to improve visualisation. A shaver or mini grasper is used for removal of fracture haematoma and osteocartilaginous debris. Cartilage damage will be graded using the Outerbridge classification system [22] (Additional file 3). With the 1-mm hook probe, assessment of the quality of reduction and ligamentous injuries will be performed. Step-off and gaps will be measured with a calibrated 1-mm probe at the point of maximum displacement and recorded. The trampoline and hook test are performed to demonstrate a triangular fibrocartilage complex (TFCC) tear. TFCC tears will be classified according to Palmer [23] (Additional file 4). All scapholunate ligament injuries will be noted and graded according to the Geissler classification [11] (Additional file 5).




The same classification will be applied for lunotriquetral injuries. Wound closure will be performed using standard techniques. All patients will receive a pressure bandage for 24-48 h.

For both the intervention and the control group, patients are allowed to start exercising immediately after the operation. Exercises include pronation and supination, flexion and extension, and ulnar and radial deviation of the wrist. Patients are instructed to use the affected extremity as far as pain allows. However, only non-weight-bearing practice is allowed for the first six weeks. Rehabilitation with the assistance of a physiotherapist is recommended at the discretion of the patient and treating surgeon.

All interventions are performed according to predefined Standard Operating Procedures (SOPs). Individuals can leave the study at any time for any reason if they wish to do so without any consequences. The investigator can decide to withdraw a participant from the study for urgent medical reasons.

\section{Outcomes}

\section{Primary outcome}

The primary outcome of this study is wrist pain and disability expressed as change on the PRWE score after three months. In addition, the PRWE questionnaire will be completed after three and six weeks, and six and 12 months of follow-up (Fig. 2). The PRWE is a validated tool for assessing functional outcome in patients with distal radius fractures $[24,25]$. The PRWE is a 15 -item questionnaire which measures wrist pain and disability in activities of daily living on a scale of $0-10$. Although the PRWE consists of three subscales (pain, function and cosmetics), the PRWE results in a single score [26]. The highest score, indicating severe impairment, is 100 and the best score, indicating no impairment, is zero. The Dutch version has been structurally validated [26]. The PRWE score will be expressed as a final value at each of the follow-up moments.

\section{Secondary outcomes}

- Wrist function, disability and pain as measured with the DASH score, at three and six weeks and three, six and 12 months of follow-up (Fig. 2). The DASH questionnaire is a 30-item, self-report questionnaire which measures physical function and symptoms in patients with any or several musculoskeletal disorders of the upper limb, including the distal radius [27-29]. The DASH questionnaire tests the degree of difficulty in performing a variety of physical activities because of arm, shoulder or hand problems (six items), the severity of pain, tingling (two items), as well as the effect of the upper limb problem on social activities, work and sleep (three items). The highest score is 100, indicating severe disability and pain; the lowest score

\begin{tabular}{|c|c|c|c|c|c|c|c|c|c|c|}
\hline \multirow[b]{3}{*}{ TIMEPOINT } & \multirow{3}{*}{$\begin{array}{c}\text { Enrolment } \\
-t_{1} \\
\end{array}$} & \multirow{3}{*}{$\begin{array}{c}\text { Allocation } \\
0 \\
\end{array}$} & \multicolumn{8}{|c|}{ STUDY PERIOD } \\
\hline & & & \multirow[b]{2}{*}{ Baseline } & \multicolumn{7}{|c|}{ Post-allocation } \\
\hline & & & & $\begin{array}{c}\text { Day } \\
1\end{array}$ & $\begin{array}{c}\text { Week } \\
1\end{array}$ & $\begin{array}{c}\text { Week } \\
3\end{array}$ & $\begin{array}{c}\text { Week } \\
6\end{array}$ & \begin{tabular}{|c} 
Month \\
3
\end{tabular} & $\begin{array}{c}\text { Month } \\
6\end{array}$ & $\begin{array}{c}\text { Month } \\
12 \\
\end{array}$ \\
\hline \multicolumn{11}{|l|}{ ENROLMENT: } \\
\hline \multirow{2}{*}{$\begin{array}{l}\text { Eligibility screen } \\
\text { Informed consent }\end{array}$} & $x$ & & & & & & & & & \\
\hline & $\mathrm{x}$ & & & & & & & & & \\
\hline Allocation & & $\mathrm{x}$ & & & & & & & & \\
\hline \multicolumn{11}{|l|}{ INTERVENTIONS: } \\
\hline $\begin{array}{l}\text { ORIF + wrist } \\
\text { arthroscopy }\end{array}$ & & & $\mathrm{x}$ & & & & & & & \\
\hline ORIF & & & $\mathrm{x}$ & & & & & & & \\
\hline \multicolumn{11}{|l|}{ ASSESSMENTS: } \\
\hline Baseline variables & & $\mathrm{x}$ & & & & & & & & \\
\hline PRWE & & & & & & $\mathrm{x}$ & $\mathrm{x}$ & $\mathrm{x}$ & $\mathrm{x}$ & $\mathrm{x}$ \\
\hline DASH & & & & & & $\mathrm{x}$ & $\mathrm{x}$ & $\mathrm{x}$ & $\mathrm{x}$ & $\mathrm{x}$ \\
\hline VAS & & & & $\mathrm{x}$ & $\mathrm{x}$ & $\mathrm{x}$ & $\mathrm{x}$ & $\mathrm{x}$ & & \\
\hline \multirow{2}{*}{$\begin{array}{r}\text { Range of motion } \\
\text { Grip strength }\end{array}$} & & & & & & $\mathrm{x}$ & $\mathrm{x}$ & $\mathrm{x}$ & & \\
\hline & & & & & & $x$ & $\mathrm{x}$ & $\mathrm{x}$ & & \\
\hline Cost-effectiveness & & & & & & $x$ & $x$ & $\mathrm{x}$ & & \\
\hline
\end{tabular}

Fig. 2 Follow-up visits 
is zero, indicating no disability and pain. The Dutch version of the DASH questionnaire has been validated and has shown to be a reliable and valid instrument [30]. The DASH score will be expressed as a final value at each of the follow-up moments.

- Postoperative pain as indicated on a Visual Analogue Scale (VAS), where zero means no pain and ten the worst pain possible. Patients will be asked to give an estimation of their pain and the type and quantity of pain medication taken postoperatively at one day, one week, three weeks, six weeks and three months (Fig. 2). The VAS pain score will be expressed as the final value at each of the follow-up moments.

- ROM of the wrist measured on both the injured as well as the uninjured wrist with a handheld goniometer. Measurements of ROM include ulnar and radial deviation, pronation and supination, and flexion and extension of the wrist. ROM is measured at three weeks, six weeks and three months, and will be expressed as both a final value and as a percentage of the uninjured side (Fig. 2).

- Prehensile grip strength as a percentage of the uninjured wrist. Grip strength will be measured on both sides with a Baseline dynamometer (White Plains, NY, USA) with the arm of the patient to the side and the elbow at $90^{\circ}$ flexion. Grip strength will be calculated as the mean of three measurements and expressed as a final value and as a percentage of the uninjured side. Grip strength is measured at three weeks, six weeks and three months (Fig. 2).

- Complications, such as superficial or deep infection divided by the criteria of the US Centers for Disease Control and Prevention [31], tendinitis or rupture of one of the flexor or extensor tendons, carpal tunnel syndrome, compartment syndrome, complex regional pain syndrome (CRPS) type 1 according to the Veldman [32] and the Budapest criteria [33, 34], and hardware-related complications will be recorded.

- Cost-effectiveness and cost-utility of ORIF with and without an arthroscopic-assisted procedure from a societal perspective, measured with an economic evaluation questionnaire at three weeks, six weeks and three months follow-up. The economic evaluation questionnaire is based on the EQ-5D and the Standard Form Health and Labour questionnaire. The EQ-5D will be used to measure qualityadjusted life years (QALY). Since this analysis is from a societal perspective, direct healthcare costs, direct non-healthcare costs and indirect costs due to the operative treated distal radius fracture will be considered (Table 1). A more detailed description of the economic analysis can be found in the protocol of the VIPAR trial [35].
Table 1 Costs included in the economic evaluation

Direct healthcare costs
Open reduction and internal fixation
Additional cost of wrist arthroscopy
Follow-up visits medical specialist
Additional visits to healthcare professional
Prescribed medication
Professional home care
Treatment and follow-up of complications
Physical therapy
Direct non-healthcare costs
Travel expenses to and from the hospital
Over the counter medication
Care provided by family or paid help
Assistive devices
Indirect costs

Absenteeism from paid labour (per day)

The cost-effectiveness is determined at three weeks, six weeks and three months (Fig. 2).

- In the intervention group the quality of reduction, associated ligamentous injuries and cartilage damage will be assessed. Ligamentous injuries are divided in TFCC injuries, classified according to the Palmer classification [23], and scapholunate ligament and lunotriquetral injuries, graded according to the Geissler classification [11].

\section{Randomisation}

All consecutive adult patients who are diagnosed with a displaced complete articular distal radius fracture $(\mathrm{AO} /$ OTA type C) and scheduled for ORIF will be invited to participate in this study if they meet the inclusion and exclusion criteria. Informed consent will be obtained at the outpatient clinic before the operation. Randomisation will be performed by means of a computerised randomisation procedure, using Castor ${ }^{\circledR}$, which is an online secure randomisation service. Allocation concealment will be ensured until patients have been randomised, which takes place after baseline characteristics have been obtained. The sequence of allocation is concealed until trial completion. To avoid imbalances between treatment groups, patients will be randomised in two strata according to age: $18-65$ years and $\geq 65$ years using a mixed block randomisation with blocks of four, six and eight patients. The order of the block sizes is unknown to the researchers, who therefore remain blinded to the allocation of the next individual throughout the whole study. 


\section{Blinding}

Since the treatment allocation involves a surgical procedure and therefore the surgical incision and portal entrees will be visible for both physician and patient, randomisation status will not be blinded.

\section{Sample size calculation}

The sample size calculation is based on our primary outcome, the PRWE score. We choose the PRWE score at three months as our primary outcome, since we expect patients to profit most from additional wrist arthroscopy within three months after the initial trauma. After this point, the haematoma has dissolved without intervention. The mean PRWE score after a distal radius fracture after three months of follow-up in adult patients is 28 with a standard deviation of 21.3 [36]. This PRWE score was measured in a population in which $38 \%$ of patients suffered from a complete articular distal radius fracture (AO/OTA type $\mathrm{C}$ fracture). Although this cohort of patients is not fully comparable to our cohort of patients, it is the data which most closely resembles our study population. We chose an effect size of 18 points on the PRWE score at three months, since we expect the greatest difference in PRWE score between both groups at three months of follow-up. The minimally clinically important difference is set at 11.5, therefore every difference $>11.5$ is clinically meaningful [37]. Therefore, at $\alpha$ $=0.05 \%$ and a power of $80 \%$, we would require 46 patients in total and 23 per treatment arm. For safety measures and with an expected loss to follow-up of 5\%, 25 patients in each arm will be included. In a separate study conducted in the Netherlands by our research group, a prevalence of AO/OTA type $\mathrm{C}$ distal radius fractures of approximately $25 \%$ was found [4]. Therefore, we estimate to include and follow-up all 50 patients in a maximum of 1.5 years.

\section{Data analysis}

All patients will be analysed according to the intentionto-treat protocol. General descriptive statistics on patient characteristic at baseline will be performed including factors such as gender and age, and presented as percentages (categorical variables) or means and standard deviation (SD) (continuous variables), whichever is applicable. Normality will be determined by visually inspecting the plotted data distribution in a histogram. Differences between the two groups in the primary outcome, the PRWE score, will be analysed using an analysis of covariance (ANCOVA), corrected for age. The same applies for the DASH score at the different followup moments. The secondary outcomes-pain (VAS), ROM and grip strength-will be analysed using a linear mixed model. The best covariance structure for each linear mixed model is determined using the smallest
Akaike information criterion (AIC). The VAS pain score will be corrected for painkiller use. Differences in complication rates between the two treatment groups will be analysed using the Chi-square test or Fisher's exact test (in case the expected incidence is less than five). Subgroup analyses will be performed on gender and age. Multiple imputation will be used in case of $>10 \%$ missing data.

\section{Data management and monitoring}

All follow-up moments are part of the regular outpatient clinic appointments. Data of patients lost to follow-up will be analysed until the last follow-up appointment. Data will be stored in two separate files. One dataset will contain coded patient information, based on an unambiguous identification code, and a second set of medical history linked to these codes. The coordinating investigator safeguards the key to the code. The same applies for all screened patients. Data are entered in Castor ${ }^{\oplus}$. All entered data and changes are saved; a list is maintained of all individuals who are authorised to make data changes. A reason is always indicated when changes are made to the data. All data are adequately backed up and can be retrieved form the archive. All researchers involved in the study will have access to all data collected. Data will be stored and kept for 15 years according standard guidelines.

The Institutional Review Board waived the need for a data monitoring committee, since both treatment modalities are part of standard care. An audit is performed half way during the trial.

\section{Protocol amendments}

For any modifications of the study protocol (29 December 2016; version 6) that may impact the study, approval will be obtained from the Institutional Review Board before implementation. Protocol modifications are communicated to relevant parties by letter.

\section{Adverse events}

All adverse events will be described in the patient file during consult at any of the follow-up visits or any other moment if indicated or requested by the patient. This includes wound infection, complex regional pain syndrome, compartment syndrome and any neurovascular or tendon damage. Complex regional pain syndrome will be classified according to the 'Budapest Criteria' created and validated by the Budapest consensus group [33, 34].

All serious adverse events (SAE) are reported to the accredited medical ethics board that approved the protocol, within 15 days after the sponsor has first knowledge of the serious adverse reactions. Arthroscopic-related complications which require a readmission or reoperation are listed in a periodic overview. 
SAEs that result in death or are life-threatening should be reported expeditiously. The expedited reporting will occur not later than seven days after the responsible investigator has first knowledge of the adverse reaction. This is for a preliminary report with another eight days for completion of the report.

All adverse events will be followed until they have abated or until a stable situation has been reached. Depending on the event, follow-up may require additional tests or medical procedures as indicated, and/or referral to the general physician or a medical specialist.

\section{Ethics}

This study will be conducted according to the principles of the Declaration of Helsinki (64th WMA General Assembly, Fortaleza, Brazil, October 2013) and in accordance with the Medical Research Involving Human Subjects Act (WMO) and 'Good Clinical Practice' guidelines. Insurance was set up for compensation for the study participants who suffer from potential harm.

\section{Dissemination policy}

The results of this study will be submitted for publication in a peer-reviewed journal. The criteria for authorship will follow the guidelines established by the International Committee of Medical Journal Editors.

\section{Discussion}

Randomisation status will not be blinded, since the treatment allocation involves a surgical procedure and therefore the surgical incision and the portal entrees are visible for both physician and patient.

\section{Trial status}

This trial has finished recruiting patients.

\section{Additional files}

Additional file 1: Participating centres. (DOC $23 \mathrm{~kb}$ )

Additional file 2: SPIRIT 2013 checklist. Standard Protocol Items: Recommendations for Interventional Trials. (DOC $123 \mathrm{~kb}$ )

Additional file 3: Outerbridge classification for cartilage damage. (PDF $191 \mathrm{~kb}$ )

Additional file 4: Palmer classification for TFCC acute traumatic tears. (PDF $118 \mathrm{~kb}$ )

Additional file 5: Geissler classification for SL and LT tears. (PDF 106 kb)

\section{Abbreviations}

AO/OTA: Arbeitsgemeinshaft für Osteosynthesefragen/Orthopedic Trauma Association; CT: Computed tomography; DASH: Disability of the Arm, Shoulder and Hand; ISS: Injury Severity Score; MCR: Midcarpal radial; MCU: Midcarpal ulnar; ORIF: Open reduction and internal fixation; PA: Posterior-anterior; PRWE: Patient-Rated Wrist Evaluation; ROM: Range of motion; SAE: Serious adverse event; SD: Standard deviation; VAS: Visual Analogue Scale

\section{Acknowledgements}

Not applicable.

\section{Funding}

An unrestricted start-up grant was received from Foundation Coolsingel for the participants' insurance (457). The protocol has undergone peer review as part of the funding application. There was no involvement of this organisation in the design of this study and preparation of this manuscript. No additional funding was received.

\section{Availability of data and materials}

The datasets generated, used and analysed during the current study are available from the corresponding author upon reasonable request.

\section{Authors' contributions}

All authors participated in the design of the study and the drafting of the manuscript. All authors have read and approved the final manuscript. All authors will have access to all data collected. MAMM and NWLS participated in the design of the study and drafted the manuscript, and made and will make a substantial contribution to the acquisition of the data. CAS, JWC, RWP and BIC helped to draft and critically revise the manuscript, and made and will make a substantial contribution to the acquisition of the data.

\section{Ethics approval and consent to participate}

The Institutional Review Board of the Maasstad Hospital in Rotterdam has approved the protocol on 28 October 2015 and approved the participating centres to conduct the study (NL54377.101.15). The board of directors of the Maasstad Hospital (L2015-75), Erasmus Medical Center (MEC-2016-372) and the Academic Medical Center (2016_263\#A2016107) approved local feasibility on 21 December 2015, 22 June 2016 and 11 January 2017, respectively. Patients' informed consent will be obtained from all participants.

\section{Consent for publication}

Not applicable in this section.

\section{Competing interests}

The authors declare that they have no competing interests.

\section{Publisher's Note}

Springer Nature remains neutral with regard to jurisdictional claims in published maps and institutional affiliations.

\section{Author details}

${ }^{1}$ Trauma Unit, Department of Surgery, Academic Medical Center, University of Amsterdam, P.O. Box 22660, 1100 DD Amsterdam, The Netherlands. ${ }^{2}$ Department of Orthopaedic Surgery, Erasmus Medical Center, P.O. Box 2040, 3000 CA Rotterdam, The Netherlands. ${ }^{3}$ Department of Surgery, Maasstad Hospital, P.O. Box 9100, 3007 AC Rotterdam, The Netherlands.

Received: 19 January 2017 Accepted: 18 December 2017

Published online: 02 February 2018

References

1. Koval KJ, Harrast JJ, Anglen JO, Weinstein JN. Fractures of the distal part of the radius. The evolution of practice over time. Where's the evidence? J Bone Joint Surg Am. 2008;90:1855-61.

2. Schneppendahl J, Windolf J, Kaufmann RA. Distal radius fractures: current concepts. J Hand Surg [Am]. 2012;37:1718-25.

3. Mattila VM, Huttunen TT, Sillanpaa P, Niemi S, Pihlajamaki H, Kannus P. Significant change in the surgical treatment of distal radius fractures: a nationwide study between 1998 and 2008 in Finland. J Trauma. 2011;71: 939-42. discussion 942-933.

4. Bentohami A, Bosma J, Akkersdijk GJM, van Dijkman B, Goslings JC, Schep NWL. Incidence and characteristics of distal radial fractures in an urban population in The Netherlands. Eur J TraumaEmerg Surg. 2014;40:357-61.

5. Arora R, Lutz M, Deml C, Krappinger D, Haug L, Gabl M. A prospective randomized trial comparing nonoperative treatment with volar locking plate fixation for displaced and unstable distal radial fractures in patients sixty-five years of age and older. J Bone Joint Surg Am. 2011;93:2146-53. 
6. Bartl C, Stengel D, Bruckner T, Gebhard F. The treatment of displaced intra-articular distal radius fractures in elderly patients. Dtsch Arztebl Int. 2014;111:779-87.

7. Ruch DS, Vallee J, Poehling GG, Smith BP, Kuzma GR. Arthroscopic reduction versus fluoroscopic reduction in the management of intra-articular distal radius fractures. Arthroscopy. 2004;20:225-30.

8. Auge 2nd WK, Velazquez PA. The application of indirect reduction techniques in the distal radius: the role of adjuvant arthroscopy. Arthroscopy. 2000;16:830-5.

9. Edwards 2nd CC, Haraszti CJ, McGillivary GR, Gutow AP. Intra-articular distal radius fractures: arthroscopic assessment of radiographically assisted reduction. J Hand Surg [Am]. 2001;26:1036-41.

10. Ono H, Furuta K, Fujitani R, Katayama T, Akahane M. Distal radius fracture arthroscopic intraarticular displacement measurement after open reduction and internal fixation from a volar approach. J Orthop Sci. 2010;15:502-8.

11. Geissler WB, Freeland AE, Savoie FH, Mclntyre LW, Whipple TL. Intracarpal soft-tissue lesions associated with an intra-articular fracture of the distal end of the radius. J Bone Joint Surg Am. 1996;78:357-65.

12. Peicha G, Seibert F, Fellinger M, Grechenig W. Midterm results of arthroscopic treatment of scapholunate ligament lesions associated with intra-articular distal radius fractures. Knee Surg Sports Traumatol Arthrosc. 1999;7:327-33

13. Ruch DS, Yang CC, Smith BP. Results of acute arthroscopically repaired triangular fibrocartilage complex injuries associated with intra-articular distal radius fractures. Arthroscopy. 2003;19:511-6.

14. Lindau T, Arner M, Hagberg L. Intraarticular lesions in distal fractures of the radius in young adults. A descriptive arthroscopic study in 50 patients. J Hand Surg Br. 1997;22:638-43.

15. Vener MJ, Thompson Jr RC, Lewis JL, Oegema Jr TR. Subchondral damage after acute transarticular loading: an in vitro model of joint injury. J Orthop Res. 1992;10:759-65.

16. Mrkonjic A, Geijer M, Lindau T, Tagil M. The natural course of traumatic triangular fibrocartilage complex tears in distal radial fractures: a 13-15 year follow-up of arthroscopically diagnosed but untreated injuries. J Hand Surg [Am]. 2012;37:1555-60.

17. Yamazaki H, Uchiyama S, Komatsu M, Hashimoto S, Kobayashi Y, Sakurai T, et al. Arthroscopic assistance does not improve the functional or radiographic outcome of unstable intra-articular distal radial fractures treated with a volar locking plate: a randomised controlled trial. Bone Joint J. 2015:97-b:957-62.

18. Chan AW, Tetzlaff JM, Altman DG, Laupacis A, Gotzsche PC, Krle AJK, et al. SPIRIT 2013 Statement: defining standard protocol items for clinical trials. Rev Panam Salud Publica. 2015;38:506-14.

19. Brink PRG, Bransz N, Deijkers RLM, van Eerten PV, Kolkman S, van Loon J, et al. Guideline distal radius fractures, diagnosis and treatment. 2010. https://heelkunde.nl/sites/heelkunde.nl/files/richtlijnen-definitief/Richtlijn Distale_radius_fracturen_definitieve_versie_0511.pdf. Accessed 15 Dec 2016

20. Ono H, Katayama T, Furuta K, Suzuki D, Fujitani R, Akahane M. Distal radial fracture arthroscopic intraarticular gap and step-off measurement after open reduction and internal fixation with a volar locked plate. J Orthop Sci. 2012;17:443-9.

21. Henry MH, Griggs SM, Levaro F, Clifton J, Masson MV. Volar approach to dorsal displaced fractures of the distal radius. Tech Hand Up Extrem Surg. 2001:5:31-41.

22. Outerbridge RE. The etiology of chondromalacia patellae. Clin Orthop Relat Res. 2001;1961:5-8.

23. Palmer AK. Triangular fibrocartilage complex lesions: a classification. J Hand Surg [Am]. 1989;14:594-606.

24. Changulani M, Okonkwo U, Keswani T, Kalairajah Y. Outcome evaluation measures for wrist and hand: which one to choose? Int Orthop. 2008;32:1-6.

25. MacDermid JC, Turgeon T, Richards RS, Beadle M, Roth JH. Patient rating of wrist pain and disability: a reliable and valid measurement tool. J Orthop Trauma. 1998;12:577-86.

26. El Moumni M, Van Eck ME, Wendt KW, Reininga IH, Mokkink LB. Structural validity of the Dutch version of the Patient-Rated Wrist Evaluation (PRWE-NL) in patients with hand and wrist injuries. Phys Ther. 2016;96:908-16.

27. Brogren E, Hofer M, Petranek M, Wagner P, Dahlin LB, Atroshi I. Relationship between distal radius fracture malunion and arm-related disability: a prospective population-based cohort study with 1-year follow-up. BMC Musculoskelet Disord. 2011;12:9.

28. Calfee RP, Adams AA. Clinical research and patient-rated outcome measures in hand surgery. J Hand Surg [Am]. 2012;37:851-5.
29. Hoang-Kim A, Pegreffi F, Moroni A, Ladd A. Measuring wrist and hand function: common scales and checklists. Injury. 2011:42:253-8.

30. Raven EE, Haverkamp D, Sierevelt IN, van Montfoort DO, Poll RG, Blankevoort L, et al. Construct validity and reliability of the disability of arm, shoulder and hand questionnaire for upper extremity complaints in rheumatoid arthritis. J Rheumatol. 2008;35:2334-8.

31. Mangram AJ, Horan TC, Pearson ML, Silver LC, Jarvis WR. Guideline for prevention of surgical site infection, 1999. Hospital Infection Control Practices Advisory Committee. Infect Control Hosp Epidemiol. 1999;1999(20):250-78. quiz 279-280.

32. Veldman PHJM, Reynen HM, Arntz IE, Goris RJA. Signs and symptoms of reflex sympathetic dystrophy: Prospective study of 829 patients. Lancet. 1993;342:1012-6.

33. Harden RN, Bruehl S, Perez RS, Birklein F, Marinus J, Maihofner C, et al. Validation of proposed diagnostic criteria (the "Budapest Criteria") for complex regional pain syndrome. Pain. 2010;150:268-74.

34. Harden RN, Bruehl S, Stanton-Hicks M, Wilson PR. Proposed new diagnostic criteria for complex regional pain syndrome. Pain Med. 2007;8:326-31.

35. Mulders MA, Walenkamp MM, Goslings JC, Schep NW. Internal plate fixation versus plaster in displaced complete articular distal radius fractures, a randomised controlled trial. BMC Musculoskelet Disord. 2016;17:68.

36. MacDermid JC, Roth JH, Richards RS. Pain and disability reported in the year following a distal radius fracture: a cohort study. BMC Musculoskelet Disord. 2003:4:24

37. Walenkamp MM, de Muinck Keizer RJ, Goslings JC, Vos LM, Rosenwasser MP Schep NW. The minimum clinically important difference of the patient-rated wrist evaluation score for patients with distal radius fractures. Clin Orthop Relat Res. 2015;473:3235-41.

\section{Submit your next manuscript to BioMed Central and we will help you at every step:}

- We accept pre-submission inquiries

- Our selector tool helps you to find the most relevant journal

- We provide round the clock customer support

- Convenient online submission

- Thorough peer review

- Inclusion in PubMed and all major indexing services

- Maximum visibility for your research

Submit your manuscript at www.biomedcentral.com/submit
) Biomed Central 